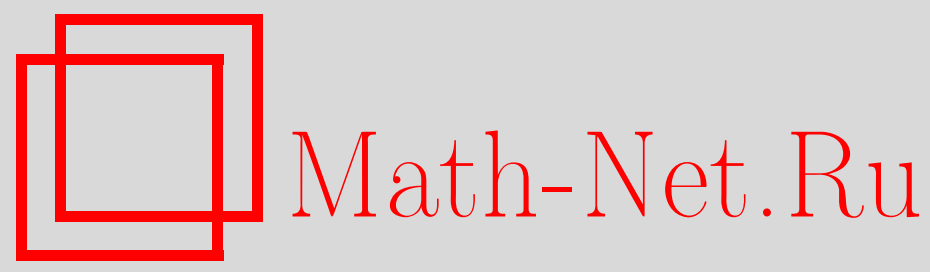

В. И. Солодовников, Гомоморфизмы регистров сдвига в линейные автоматы, Дискрет. матем., 2008, том 20, выпуск 4, 89-101

DOI: https://doi.org/10.4213/dm1029

Использование Общероссийского математического портала Math-Net.Ru подразумевает, что вы прочитали и согласны с пользовательским соглашением http://www . mathnet.ru/rus/agreement

Параметры загрузки:

IP: 54.210 .77 .194

26 апреля 2023 г., 15:41:11 


\title{
Гомоморфизмы регистров сдвига в линейные автоматы
}

\author{
() 2008 г. $\quad$ В. И. Солодовников
}

\begin{abstract}
Работа посвящена описанию всех гомоморфизмов регистров сдвига над конечными полями с подстановочной по входной переменной функцией обратной связи в линейные автоматы. Доказывается, что линейный автомат, который является гомоморфным образом регистра сдвига, изоморфен линейному регистру сдвига. Тем самым по ранее полученной автором теореме вопрос о гомоморфизмах регистров сдвига в линейные автоматы сводится к вопросу о разложении функции (или представляющего ее многочлена) в так называемую сдвиг-композицию двух функций (многочленов), из которых левая функция является аффинной. Доказывается также, что всякий многочлен однозначно представляется в виде суммы сдвиг-композиций линейных многочленов и одночленов с первой переменной. Этим линейным многочленам ставятся в соответствие многочлены от одной переменной, и вопрос о разложении сводится к поиску общих делителей последних. Указываются некоторые простые условия, достаточные для отсутствия нетривиальных внутренних гомоморфизмов регистра сдвига в линейные автоматы.
\end{abstract}

Гомоморфизмы являются неотъемлемой частью любой категории, в том числе и категории автоматов. Традиционный для теории автоматов интерес представляют автоматы, называемые регистрами сдвига. Это автоматы, вырабатывающие управляемые (то есть в общем случае зависящие от входа) усложненные (некоторой выходной функцией) рекуррентные последовательности. В [2] доказано, что всякий гомоморфизм двоичного регистра сдвига с линейной по входной переменной функцией обратной связи разлагается в композицию гомоморфизма на регистр и некоторого гомоморфизма, близкого к изоморфизму. При описании этого разложения основную роль играет некая бинарная некоммутативная операция на функциях (называемая здесь сдвиг-композицией). Она в некотором смысле распространяет операцию умножения многочленов от одной переменной на множество всех функций. Вопрос о гомоморфизмах регистра сводится к вопросу о поиске общих правых делителей его функции обратной связи и выходной функции.

Настоящая работа посвящена описанию всех гомоморфизмов регистров сдвига над конечными полями с подстановочной по входной переменной функцией обратной связи в линейные автоматы.

В работе приняты следующие обозначения и терминология.

Пусть $X-$ произвольное конечное множество, $F_{n+1}(X)=\left\{f \mid f: X^{n+1} \rightarrow X\right\}-$ множество всех $X$-значных функций от $n+1$ переменной, $n \geqslant 0, F(X)=\bigcup_{n=0}^{\infty} F_{n+1}(X)$.

Функцию $f \in F_{n+1}(X)$ называют подстановочной (биективной) по $i$-й переменной, если при любой фиксации всех остальных переменных получаемое отображение $X \rightarrow X$ 
является биекцией. В случае подстановочности по последней, то есть по $(n+1)$-й переменной, через $f^{-1}$ будем обозначать функцию, обратную к функции $f$ по последней переменной, то есть $f^{-1} \in F_{n+1}(X), f^{-1}\left(x_{1}, \ldots, x_{n}, f\left(x_{1}, \ldots, x_{n+1}\right)\right)=x_{n+1}$. В двоичном случае подстановочность по какой-либо переменной равносильна линейности по этой переменной.

Через $F_{n+1}^{*}(X)$ обозначим множество всех подстановочных по последней переменной функций из $F_{n+1}(X), F^{*}(X)=\bigcup_{n=0}^{\infty} F_{n+1}^{*}(X)$.

Через $P=G F(q)$ обозначим конечное поле из $q$ элементов, $P[x]-$ кольцо многочленов над $P$ от одной переменной $x, d(\varphi)-$ степень многочлена $\varphi$.

Пусть $M(P)$ - множество всех многочленов над $P$ от переменных $\left\{x_{i}, i \geqslant 1\right\}$ с ограниченными степенями, то есть формул вида

$$
g=\sum_{0 \leqslant k, 1 \leqslant i_{1}<\ldots<i_{k}, 1 \leqslant s_{j} \leqslant q-1} a_{i_{1}, \ldots, i_{k} ; s_{1}, \ldots, s_{k}} x_{i_{1}}^{s_{1}} \ldots x_{i_{k}}^{s_{k}},
$$

где $a_{i_{1}, \ldots, i_{k} ; s_{1}, \ldots, s_{k}} \in P$ называют коэффициентом при одночлене $x_{i_{1}}^{s_{1}} \ldots x_{i_{k}}^{s_{k}}$ степени $s_{1}+\ldots+s_{k}$ и только конечное число коэффициентов отлично от нуля. Говорят, что одночлен входит в $g$, если коэффициент при нем ненулевой. Максимальная степень входящих в ненулевой многочлен $g$ одночленов называется степенью $g$ и обозначается через $d(g)$. Номером одночлена $x_{i_{1}}^{s_{1}} \ldots x_{i_{k}}^{s_{k}}$ назовем число

$$
\left|x_{i_{1}}^{s_{1}} \ldots x_{i_{k}}^{s_{k}}\right|=\sum_{j=1}^{k} s_{j} q^{i_{j}-1} .
$$

Каждый одночлен однозначно определяется своим номером. Хорошо известно, что любая функция из $F(P)$ однозначно представляется некоторым многочленом из $M(P)$. В случаях, когда число переменных функции определено, будем при необходимости отождествлять эту функцию с представляющим ее многочленом.

Пусть $M^{*}(P)$ - множество всех многочленов из $M(P)$ положительной степени, подстановочных (как функции) по последней (то есть существенной с максимальным номером) переменной.

Автоматом с входным алфавитом $X$, множеством состояний $S$, выходным алфавитом $Y$, функцией переходов $\delta: S \times X \rightarrow S$ и функцией выходов $\lambda: S \times X \rightarrow Y$ называют пятерку объектов $A=(X, S, Y, \delta, \lambda)$. При этом подразумевается, что если автомат $A$ находится в состоянии $s \in S$ и на его вход подается входной символ $x \in X$, то на его выходе появляется выходной символ $\lambda(s, x)$ и автомат переходит в состояние $\delta(s, x)$.

Для отображений $\psi$ и $\varphi$ через $\psi \varphi$ обозначается их композиция, при которой $\varphi$ действует первым. Аналогично, для автоматов $A$ и $B$ через $A B$ обозначается их последовательное соединение, при котором выход с автомата $B$ подается на вход автомата $A$.

Гомоморфизмом автомата $A^{\prime}=\left(X^{\prime}, S^{\prime}, Y^{\prime}, \delta^{\prime}, \lambda^{\prime}\right)$ в автомат $A=(X, S, Y, \delta, \lambda)$ называется тройка отображений $(\alpha, \beta, \gamma), \alpha: X^{\prime} \rightarrow X, \beta: S^{\prime} \rightarrow S, \gamma: Y^{\prime} \rightarrow Y$, удовлетворяющих условиям $\beta \delta^{\prime}=\delta(\beta \times \alpha), \gamma \lambda^{\prime}=\lambda(\beta \times \alpha)$. В этом случае пишут $(\alpha, \beta, \gamma): A^{\prime} \rightarrow A$. Если $\alpha$ и $\gamma$ - тождественные вложения, то гомоморфизм называется внутренним и обозначается $\beta: A^{\prime} \rightarrow A$.

Любой гомоморфизм $(\alpha, \beta, \gamma): A^{\prime} \rightarrow A$ сводится к внутреннему гомоморфизму $\beta: \gamma A^{\prime} \rightarrow A \alpha$, а в случае биективности $\alpha$ и к внутреннему гомоморфизму $\beta: \gamma A^{\prime} \alpha^{-1} \rightarrow A$. Поэтому часто достаточно рассматривать только внутренние гомоморфизмы.

Композиция гомоморфизмов (покоординатная композиция троек отображений) также является гомоморфизмом. 

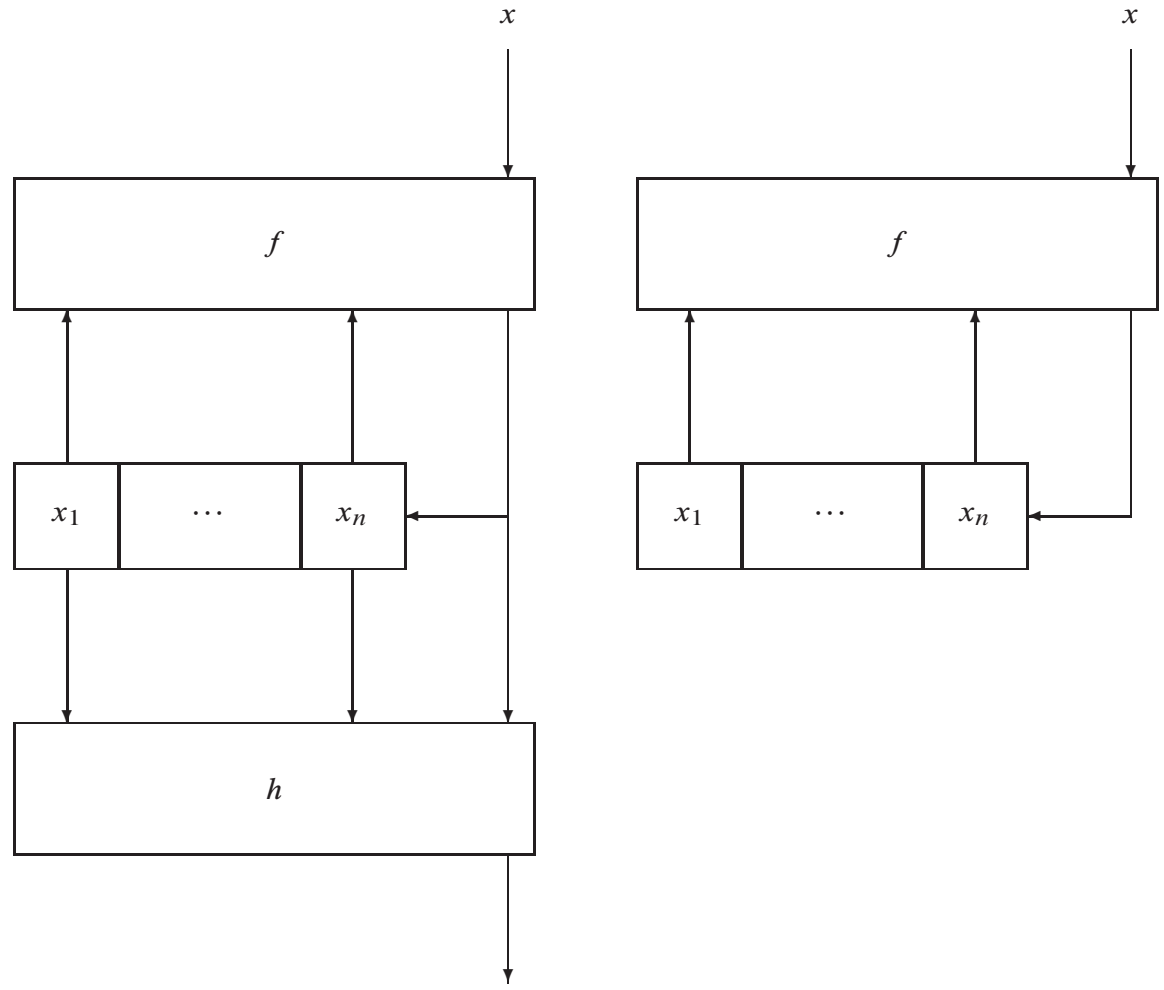

$R(h, f)$

$R(f)$

Рис. 1.

Гомоморфизм $(\alpha, \beta)$ автоматов без выхода назовем тривиальным, если справедливо равенство $\left|\beta\left(S^{\prime}\right)\right|=1$.

Как и в [2], автомат $R(h, f)=\left(X, X^{n}, X, \rho, \mu\right)$, у которого функция переходов $\rho$ и функция выходов $\mu$ определяются равенствами

$$
\begin{aligned}
\rho\left(\left(x_{1}, \ldots, x_{n}\right), x\right) & =\left(x_{2}, \ldots, x_{n}, f\left(x_{1}, \ldots, x_{n}, x\right)\right), \\
\mu\left(\left(x_{1}, \ldots, x_{n}\right), x\right) & =h\left(x_{2}, \ldots, x_{n}, f\left(x_{1}, \ldots, x_{n}, x\right)\right)
\end{aligned}
$$

для любых $x_{1}, \ldots, x_{n}, x \in X$, называется регистром сдвига над множеством $X$ длины $n$ с функцией обратной связи $f$ и выходной функцией $h$, где $f, h \in F_{n+1}(X)$. Регистр сдвига без выхода с функцией обратной связи $f$ обозначается через $R(f)$. Эти автоматы изображаются, как на рис. 1.

Для любых $f_{i} \in F_{n_{i}+1}(X), i=1,2$, функция $f_{2} \triangleleft f_{1} \in F_{n_{1}+n_{2}+1}(X)$ и отображение $\pi_{f_{1}, n_{2}}: X^{n_{1}+n_{2}} \rightarrow X^{n_{2}}$ определяются равенствами

$$
\begin{aligned}
f_{2} \triangleleft f_{1}\left(x_{1}, \ldots, x_{n_{1}+n_{2}+1}\right) & \\
& =f_{2}\left(f_{1}\left(x_{1}, \ldots, x_{n_{1}+1}\right), f_{1}\left(x_{2}, \ldots, x_{n_{1}+2}\right), \ldots, f_{1}\left(x_{n_{2}+1}, \ldots, x_{n_{1}+n_{2}+1}\right)\right), \\
\pi_{f_{1}, n_{2}}\left(x_{1}, \ldots, x_{n_{1}+n_{2}}\right) & \\
& =\left(f_{1}\left(x_{1}, \ldots, x_{n_{1}+1}\right), f_{1}\left(x_{2}, \ldots, x_{n_{1}+2}\right), \ldots, f_{1}\left(x_{n_{2}}, \ldots, x_{n_{1}+n_{2}}\right)\right)
\end{aligned}
$$

для любых $x_{1}, \ldots, x_{n_{1}+n_{2}+1} \in X$, так что $f_{2} \triangleleft f_{1}=f_{2} \pi_{f_{1}, n_{2}+1}$. Операцию $\triangleleft$ назовем сдвиг-композицией функций. 
Множество $F(X)$ относительно операции $\triangleleft$ является моноидом с единичным элементом $x_{1} \in F_{1}(X)$, а множество $F^{*}(X)-$ его подмоноидом. Операция $\triangleleft$ является продолжением на множество всех функций операции умножения многочленов в следующем смысле. Обозначим через $P[x]^{*}$ моноид (относительно умножения) всех ненулевых многочленов над полем $P$. Тогда отображение $w: P[x]^{*} \rightarrow F^{*}(P)$, где

$$
w\left(c_{0}+c_{1} x+\ldots+c_{n} x^{n}\right)=\left(c_{0} x_{1}+c_{1} x_{2}+\ldots+c_{n} x_{n+1}\right),
$$

является инъективным гомоморфизмом моноидов.

Следующая теорема сводит описание всех гомоморфизмов регистра на регистр с подстановочными по входной переменной функциями обратной связи к описанию разложений функций в сдвиг-композицию (см. [2]).

Теорема 1. Пусть $n_{1}, n_{2} \geqslant 0, f \in F_{n_{1}+1}^{*}(X), f_{2} \in F_{n_{2}+1}^{*}(X), h \in F_{n+1}(X)$, $h_{2} \in F_{n_{2}+1}(X), \beta: X^{n} \rightarrow X^{n_{2}}$. Тогда следующие утверждения равносильны:

(1) $\beta: R\left(h, f^{-1}\right) \rightarrow R\left(h_{2}, f_{2}^{-1}\right)-$ внутренний гомоморфизм,

(2) $n \geqslant n_{2}, \beta=\pi_{f_{1}, n_{2}}, f=f_{2} \triangleleft f_{1}, h=h_{2} \triangleleft f_{1}$ для некоторой функичи $f_{1} \in F_{n_{1}+1}^{*}(X)$, где $n_{1}=n-n_{2}$.

Далее для простоты будут рассматриваться только автоматы без выхода. Соответствующие результаты для автоматов с выходом легко получить, воспользовавшись теоремой 1.

Всюду далее $X=P-$ конечное поле.

Линейным автоматом над $P$ длины $m$ с одномерным входом без выхода назовем автомат $\left(P, P^{m}, \delta\right)$, у которого функция переходов $\delta$ определяется равенством

$$
\delta(s, x)=s Q+x b+b_{0}
$$

для всех $s \in P^{m}, x \in P$, где $Q-m \times m$ матрица над $P, b, b_{0} \in P^{m}$. Матрицу $Q$ назовем матрицей этого автомата, а вектор $b-$ его входным сдвигом.

Линейным регистром сдвига над $P$ длины $n$ без выхода называют регистр $R(f), \mathrm{y}$ которого функция $f \in F_{n+1}(P)$ представляется в виде

$$
f\left(x_{1}, \ldots, x_{n+1}\right)=a_{0}+\sum_{i=1}^{n} a_{i} x_{i}+x_{n+1} \text {. }
$$

Такие функции (многочлены) будем называть унитарными аффинными функциями (многочленами).

Ясно, что линейный регистр сдвига является сильно связными линейным автоматом. Докажем теорему, из которой следует, что других сильно связных линейных автоматов с точностью до изоморфизма нет.

Подавтомат называют внутренним, если его входной алфавит не сокращен.

Теорема 2. Для любого автомата А, являющегося внутренним подавтоматом линейного автомата над полем $P$ с одномерным входом без выхода, следующие утверждения равносильны:

(1) А является гомоморфным образом некоторого регистра сдвига над Р без выхода с подстановочной по последней (то есть входной) переменной функцией обратной связи, 
(2) А аффинно внутренне изоморфен некоторому линейному регистру сдвига над $P$ без выхода,

(3) любое состояние $A$ достижимо из некоторого состояния $A$, не меняющегося при некотором входном знаке.

Доказательство. Импликация (2) $\Rightarrow$ (1) очевидна. Докажем импликацию (1) $\Rightarrow$ (3). Из подстановочности следует, что регистр сильно связен и, например, нулевое состояние остается на месте при одном из входных знаков. Поскольку при гомоморфизме эти свойства сохраняются, (3) выполнено.

Докажем импликацию (3) $\Rightarrow(2)$. Пусть $P^{m} \subseteq S-$ множество состояний подавтомата $A$ и все они достижимы из состояния $s_{0} \in S, s_{0}=s_{0} Q+a_{0} b+b_{0}$. Тогда любое состояние из $S$ представляется в виде

$$
\delta\left(s_{0},\left(x_{1}, \ldots, x_{l}\right)\right)=s_{0}+\sum_{i=1}^{l}\left(x_{i}-a_{0}\right) b Q^{l-i} .
$$

Пусть $\sum_{i=1}^{n} a_{i} x^{i-1}+x^{n}-$ минимальный многочлен вектора $b$ относительно матрицы $Q$. Определим функцию $f \in F_{n+1}(P)$ равенством

$$
f\left(x_{1}, \ldots, x_{n+1}\right)=-a_{0}+\sum_{i=1}^{n}-a_{i} x_{i}+x_{n+1} \text {. }
$$

Пусть $e_{i}=b Q^{i-1}, i=1, \ldots, n$. Векторы $e_{1}, \ldots, e_{n}$ линейно независимы, и для любого $i \geqslant 0$ вектор $b Q^{i}$ линейно выражается через них. Поэтому $S-$ смежный класс по подпространству пространства $P^{m}$ с базисом $e_{1}, \ldots, e_{n}$.

Определим аффинное отображение $\beta: P^{n} \rightarrow S$ формулой $\beta(s)=s C E+s_{0}$, где матрицы $C$ и $E$ определяются равенствами

$$
C=\left(\begin{array}{cccccccc}
a_{2} & a_{3} & a_{4} & \ldots & a_{n-2} & a_{n-1} & a_{n} & 1 \\
a_{3} & a_{4} & a_{5} & \ldots & a_{n-1} & a_{n} & 1 & 0 \\
a_{4} & a_{5} & a_{6} & \ldots & a_{n} & 1 & 0 & 0 \\
\ldots \ldots \ldots \ldots \ldots \ldots \ldots \ldots \ldots \ldots \ldots \ldots \ldots \ldots \ldots \ldots \ldots . \\
a_{n-1} & a_{n} & 1 & \ldots & 0 & 0 & 0 & 0 \\
a_{n} & 1 & 0 & \ldots & 0 & 0 & 0 & 0 \\
1 & 0 & 0 & \ldots & 0 & 0 & 0 & 0
\end{array}\right), \quad E=\left(\begin{array}{c}
e_{2} \\
\vdots \\
e_{n}
\end{array}\right)
$$

Конструкция матрицы $C$ была предложена в доказательстве теоремы 2.1 в [1]. Поскольку матрица $C$ невырождена, $\beta$ биективно. Покажем, что $\beta: R(f) \rightarrow A-$ гомоморфизм автоматов. Нетрудно видеть, что

$$
\delta(\beta(s), x)=\left(s C E+s_{0}\right) Q+x b+b_{0}=s C\left(\begin{array}{c}
e_{2} \\
\vdots \\
e_{n} \\
-a_{1} e_{1}-\ldots-a_{n} e_{n}
\end{array}\right)+\left(x-a_{0}\right) e_{1}+s_{0}
$$




$$
\begin{aligned}
& =\left(s C\left(\begin{array}{cccccccc}
0 & 1 & 0 & 0 & \ldots & 0 & 0 & 0 \\
0 & 0 & 1 & 0 & \ldots & 0 & 0 & 0 \\
\ldots & \ldots & \ldots & \ldots \ldots & \ldots \ldots \ldots \ldots \ldots \\
0 & 0 & 0 & 0 & \ldots & 0 & 1 & 0 \\
0 & 0 & 0 & 0 & \ldots & 0 & 0 & 1 \\
-a_{1} & -a_{2} & -a_{3} & a_{4} & \ldots & a_{n-2} & a_{n-1} & -a_{n}
\end{array}\right)+\left(x-a_{0}\right)(1,0, \ldots, 0)\right) E+s_{0} \\
& \left.=\left(\begin{array}{ccccccccc}
-a_{1} & 0 & 0 & 0 & 0 & \ldots & 0 & 0 & 0 \\
0 & a_{3} & a_{4} & a_{5} & a_{6} & \ldots & a_{n-1} & a_{n} & 1 \\
0 & 0 & a_{4} & a_{5} & a_{6} & \ldots & a_{n} & 1 & 0 \\
\ldots \ldots & \ldots & \ldots & \ldots & \ldots & \ldots & \ldots & \ldots & \ldots \\
0 & a_{n-1} & a_{n} & 1 & 0 & \ldots & 0 & 0 & 0 \\
0 & a_{n} & 1 & 0 & 0 & \ldots & 0 & 0 & 0 \\
0 & 1 & 0 & 0 & 0 & \ldots & 0 & 0 & 0
\end{array}\right)+\left(x-a_{0}\right)(1,0, \ldots, 0)\right) E+s_{0} \\
& \left.=\left(\begin{array}{ccccc}
0 & 0 & \ldots & 0 & -a_{1} \\
1 & 0 & \ldots & 0 & a_{2} \\
0 & 1 & \ldots & 0 & a_{3} \\
0 & 0 & \ldots & 0 & a_{4} \\
\ldots & \ldots & \ldots & \ldots \\
0 & 0 & \ldots & 0 & a_{n-1} \\
0 & 0 & \ldots & 1 & -a_{n}
\end{array}\right) C+\left(x-a_{0}\right)(0, \ldots, 0,1) C\right) E+s_{0} \\
& \left.=\beta\left(\begin{array}{cccccc}
0 & 0 & \ldots & 0 & 0 & -a_{1} \\
1 & 0 & \ldots & 0 & 0 & -a_{2} \\
0 & 1 & \ldots & 0 & 0 & -a_{3} \\
\ldots & \ldots & \ldots & \ldots & \ldots \\
0 & 0 & \ldots & 1 & 0 & -a_{n-1} \\
0 & 0 & \ldots & 0 & 1 & -a_{n}
\end{array}\right)+\left(x-a_{0}\right)(0, \ldots, 0,1)\right) \text {. }
\end{aligned}
$$

Теорема доказана.

Следствие 1. Для любой квадратной матрищьы $Q$ над полем $P$ следующие утверждения равносильны:

(1) любой линейный автомат над полем $P$ с одномерным входом без выхода с матриией $Q$ и ненулевым входным сдвигом аффинно внутренне изоморфен некоторому линейному регистру сдвига над $Р$ без выхода,

(2) характеристический многочлен матрицы $Q$ неприводим.

Доказательство. Воспользуемся доказательством теоремы 2. Допустим, что выполнено условие (1), а (2) не выполнено. Тогда найдется ненулевой вектор $b$ с минимальным многочленом степени $n<m$. Возьмем $b_{0}=s_{0}=(0, \ldots, 0), a_{0}=0$. Тогда не все состояния достижимы из $s_{0}$, что противоречит сильной связности линейного регистра сдвига.

Наоборот, пусть выполнено условие (2). Из неприводимости следует существование состояния $s_{0} \in P^{m}$ и входного знака $a_{0} \in P$ таких, что $s_{0}=s_{0} Q+a_{0} b+b_{0}$. Минимальный многочлен делит характеристический, а вектор $b$ ненулевой, поэтому $n=m$. Следствие доказано.

Покажем, что при $q \geqslant 4$ любой линейный регистр сдвига внутренне изоморфен некоторому нелинейному. Пусть $f_{2} \in F_{n_{2}+1}^{*}(P), f_{1} \in F_{1}(P), f_{1}\left(x_{1}\right)=x_{1}^{t}, f=f_{2} \triangleleft f_{1}$. 
При $q \leqslant 4$ возьмем $t$ такое, что $(t, q-1)=1,2 \leqslant t \leqslant q-2$ (например, $t=q-2$ ). Тогда $f_{1} \in F_{1}^{*}(P)$, и по теореме 1 регистры $R\left(f^{-1}\right)$ и $R\left(f_{2}^{-1}\right)$ внутренне изоморфны, причем $f \triangleleft f_{1}^{-1}=f_{2}$. Пусть теперь $f_{2}$ - произвольная унитарная аффинная функция. Тогда $f_{2}^{-1}$ - унитарная аффинная функция, а $f$ - неаффинная функция. Но тогда и $f^{-1}$ - неаффинная функция.

Теорема 3. Пусть $n \geqslant 0, f \in F_{n+1}^{*}(P), \alpha-$ подстановка множества $P$.

(1) Если $B$ - линейный автомат над полем $P$ с одномерным входом без выхода и $(\alpha, \beta): R\left(f^{-1}\right) \rightarrow B-$ гомоморфизм, то существуют функиии $f_{1} \in F_{n_{1}+1}^{*}(P)$, $f_{2} \in F_{n_{2}+1}^{*}(P)$, где $n_{1}+n_{2}=n$, и инъективный внутренний аффинный гомоморфизм $\beta^{\prime}: R\left(f_{2}^{-1}\right) \rightarrow B$ такие, что $f \alpha^{-1} f_{2} \triangleleft f_{1}, f_{2}-$ унитарная аффинная функция u $\beta=\beta^{\prime} \pi_{f_{1}, n_{2}}$.

(2) Если $f=\alpha^{-1} f_{2} \triangleleft f_{1}$, где $f_{1} \in F_{n_{2}+1}^{*}(P), f_{2} \in F_{n_{2}+1}^{*}(P), n_{1}+n_{2}=n, f_{2}-$ унитарная аффинная функция, то $\left(\alpha, \pi_{f_{1}, n_{2}}\right): R\left(f^{-1}\right) \rightarrow R\left(f_{2}^{-1}\right)-$ гомоморфизм на линейный регистр сдвига.

Доказательство. Докажем (1). Поскольку условие (1) теоремы 2 выполнено, внутренний подавтомат $A=(\alpha, \beta)\left(R\left(f^{-1}\right)\right)$ линейного автомата $B$ внутренне аффинно изоморфен некоторому линейному регистру $R\left(f_{2}^{-1}\right)$ (то есть $f_{2}-$ унитарная аффинная функция), $f_{2} \in F_{n_{2}+1}^{*}(P)$. Пусть этот изоморфизм есть $\beta_{0}: A \rightarrow R\left(f_{2}^{-1}\right)$. Тогда условие (1) теоремы 1 выполнено для $\beta_{0} \beta: R\left(f^{-1}\right) \rightarrow R\left(f_{2}^{-1}\right) \alpha=R\left(\left(\alpha^{-1} f_{2}\right)^{-1}\right)$, и следовательно, существует функция $f_{1} \in F_{n_{1}+1}^{*}(P)$, где $n_{1}+n_{2}=n$, такая, что $\beta_{0} \beta=\pi_{f_{1}, n_{2}}$ и $f=\alpha^{-1} f_{2} \triangleleft f_{1}$. Гомоморфизм $\beta^{\prime}: R\left(f_{2}^{-1}\right) \rightarrow B$ определим равенством $\beta^{\prime}(s)=\beta_{0}^{-1}(s)$.

Утверждение (2) следует непосредственно из импликации (2) $\Rightarrow$ (1) теоремы 1 . Теорема доказана.

Теорема 3 сводит задачу об описании всех гомоморфизмов $(\alpha, \beta)$ регистра сдвига $R\left(f^{-1}\right)$ в линейные автоматы (где $\alpha-$ подстановка) к задаче о нахождении всех разложений функции $\alpha f$ в сдвиг-композицию двух функций, в которых левая функция аффинная. Оставшаяся часть статьи посвящена решению этой задачи.

Здесь целесообразно перейти с языка функций на язык представляющих их многочленов из $M(P)$.

Операцию $\triangleleft$ на $M(P)$ (сдвиг-композицию многочленов) определим следующим образом: $g_{2} \triangleleft g_{1}$ - многочлен функции $f_{2} \triangleleft f_{1}$, где $g_{i}$ - многочлен функции $f_{i}, i=1,2$.

Множество $M(P)$ относительно операций + и · есть коммутативное кольцо, а относительно операции $\triangleleft-$ моноид с единицей $x_{1}$. Отображение $F(P) \rightarrow M(P)$, ставящее в соответствие каждой функции представляющий ее многочлен, является сюръективным гомоморфизмом моноидов относительно операции $\triangleleft$.

Обозначим через $L(P)$ множество всех линейных многочленов из $M(P)$, то есть многочленов вида $a_{1} x_{1}+\ldots+a_{n} x_{n}$. Оно является коммутативным кольцом с единицей $x_{1}$ относительно операций + и $\triangleleft$, изоморфным кольцу $P[x]$, изоморфизм $v: P[x] \rightarrow L(P)$ определяется равенством

$$
v\left(c_{0}+c_{1} x_{1}+\ldots+c_{n} x^{n}\right)=c_{0} x_{1}+c_{1} x_{2}+\ldots+c_{n} x_{n+1} .
$$

Множество $M(P)$ является абелевой группой относительно сложения. Определим умножение слева многочленов из кольца $P[x]$ на многочлены из $M(P)$ равенством $\varphi g=v(\varphi) \triangleleft g$. Тогда $M(P)-$ левый $P[x]$-модуль. 
Рассмотрим одну интерпретацию модуля $M(P)$. Пусть $P[x,+]-$ аддитивная группа кольца $P[x]$. Определим на ней бинарную операцию $\times$ равенством

$$
\left(\sum_{0 \leqslant i} a_{i} x^{i}\right) \times\left(\sum_{0 \leqslant j} b_{j} x^{j}\right)=\sum_{0 \leqslant k}\left(\sum_{i \cdot j=k} a_{i} b_{j}\right) x^{k} .
$$

Легко проверить, что эта операция ассоциативна, коммутативна, дистрибутивна и $x$ - нейтральный элемент. Следовательно, множество многочленов относительно операций + и $\times$ является коммутативным кольцом с единицей $x$, которое обозначим через $P[x,+, \times]=N(P)$. Приведем некоторые очевидные его свойства.

Предложение 1. Пусть $\varphi, \psi \in N(P)$. Тогда

(1) если $d(\varphi) \geqslant 1, d(\psi) \geqslant 1, \operatorname{mo} d(\varphi \times \psi)=d(\varphi) d(\psi)$,

(2) если $\varphi, \psi \neq 0, \varphi \times \psi=0$, то либо $\varphi=a_{0}, \psi(1)=0$, либо $\psi=a_{0}, \varphi(1)=0$ для некоторого $a_{0} \in P, \alpha \neq 0$.

Следствие 2. Пусть $\varphi_{1}, \varphi_{2}, \psi \in N(P), \psi \notin P, \varphi_{1} \times \psi=\varphi_{2} \times \psi$. Тогда $\varphi_{1}-\varphi_{2} \in P$.

Определим отображение $u: P[x] \rightarrow N(P)$ равенством

$$
u\left(\sum_{0 \leqslant i} a_{i} x^{i}\right)=\sum_{0 \leqslant i} a_{i} x^{q^{i}}
$$

Нетрудно проверить, что $u$ - инъективный гомоморфизм колец. Определим умножение слева многочленов из кольца $P[x]$ на многочлены из кольца $N(P)$ равенством

$$
\varphi \bullet \psi=u(\varphi) \times \psi
$$

Тогда из гомоморфности $u$ и коммутативности $N(P)$ следует, что $N(P)-P[x]$-алгебра.

Теорема 4. Отображение $U: M(P) \rightarrow N(P)$, определяемое равенством

$$
\begin{gathered}
U\left(\sum_{0 \leqslant k, 1 \leqslant i_{1}<\ldots<i_{k}, 1 \leqslant s_{j} \leqslant-1} a_{i_{1}, \ldots, i_{k} ; s_{1}, \ldots, s_{k}} x_{i_{1}}^{s_{1}} \ldots x_{i_{k}}^{s_{k}}\right) \\
=\sum_{0 \leqslant k, 1 \leqslant i_{1}<\ldots<i_{k}, 1 \leqslant s_{j} \leqslant q-1} a_{i_{1}, \ldots, i_{k} ; s_{1}, \ldots, s_{k}} x^{\sum_{j=1}^{k} s_{j} q^{i_{j}-1}} .
\end{gathered}
$$

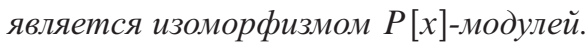

Доказательство. Члены многочлена однозначно определяются своими номерами, поэто- 
му $U-$ биекция. Так как $U\left(g_{2}+g_{1}\right)=U\left(g_{2}\right)+U\left(g_{1}\right)$ и

$$
\begin{aligned}
U(\varphi g) & =U(v(\varphi) \triangleleft g) \\
& =U\left(\left(\sum_{0 \leqslant i} a_{i} x_{i+1}\right) \triangleleft\left(\sum_{0 \leqslant k, 1 \leqslant i_{1}<\ldots<i_{k}, 1 \leqslant s_{j} \leqslant q-1} a_{i_{1}, \ldots, i_{k} ; s_{1}, \ldots, s_{k}} x_{i_{1}}^{s_{1}} \ldots x_{i_{k}}^{s_{k}}\right)\right) \\
& =U\left(\sum_{0 \leqslant k, 1 \leqslant i_{1}<\ldots<i_{k}, 1 \leqslant s_{j} \leqslant q-1} a_{i} a_{i_{1}, \ldots, i_{k} ; s_{1}, \ldots, s_{k}} x_{i_{1}+i}^{s_{1}} \ldots x_{i_{k}+i}^{s_{k}}\right) \\
& =\sum_{0 \leqslant k, 1 \leqslant i_{1}<\ldots<i_{k}, 1 \leqslant s_{j} \leqslant q-1} a_{i} a_{i_{1}, \ldots, i_{k} ; s_{1}, \ldots, s_{k}} x^{\sum_{j=1}^{k} s_{j} q^{i+i_{j}-1}} \sum_{0 \leqslant k, 1 \leqslant i_{1}<\ldots<i_{k}, 1 \leqslant s_{j} \leqslant q-1} a_{i_{1}, \ldots, i_{k} ; s_{1}, \ldots, s_{k}} x^{\sum_{j=1}^{k} s_{j} q^{i_{j}-1}} \\
& =\left(\sum_{0 \leqslant i} a_{i} x^{q^{i}}\right)_{0} U(g) .
\end{aligned}
$$

теорема доказана.

Пусть $M_{0}(P)=P \subset M(P)$, а $M_{1}(P)-$ множество всех многочленов из $M(P)$ с нулевым свободным членом, и пусть $E(P)-$ множество всех одночленов с первой переменной, то есть

$$
E(P)=\left\{x_{1}^{s_{1}} x_{i_{2}}^{s_{2}} \ldots x_{i_{k}}^{s^{k}} \mid 1 \leqslant k, 1<i_{2}<\ldots<i_{k}, 1 \leqslant s_{j} \leqslant q-1\right\} \subset M(P) .
$$

Теорема 5. $M(P)=M_{0}(P)+M_{1}(P)$ есть прямая сумма подмодулей $P[x]$-модуля $M(P)$,

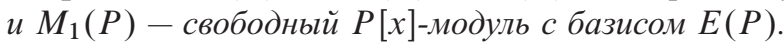

Доказательство. Первое утверждение очевидно. Поскольку

$$
x_{i_{1}}^{s_{1}} x_{i_{2}}^{s_{2}} \ldots x_{i_{k}}^{s_{k}}=x_{i_{1}} \triangleleft x_{1}^{s_{1}} x_{i_{2}-i_{1}+1}^{s_{2}} \ldots x_{i_{k}-i_{1}+1}^{s_{k}}=x^{i_{1}}\left(x_{1}^{s_{1}} x_{i_{2}-i_{1}+1}^{s_{2}} \ldots x_{i_{k}-i_{1}+1}^{s_{k}}\right),
$$

$E(P)$ порождает модуль $M_{1}(P)$. Покажем, что $E(P)-$ свободное множество. Пусть

$$
\sum_{1 \leqslant k, 1 \leqslant i_{2}<\ldots<i_{k}, 1 \leqslant s_{j} \leqslant q-1} \varphi_{i_{2}, \ldots, i_{k} ; s_{1}, \ldots, s_{k}} x_{i_{1}}^{s_{1}} \ldots x_{i_{k}}^{s_{k}}=0 .
$$

Любое натуральное число (номер одночлена) однозначно разлагается в произведение степени $q$ и некратного $q$ числа, поэтому $\varphi_{i_{2}, \ldots, i_{k} ; s_{1}, \ldots, s_{k}} x_{i_{1}}^{s_{1}} \ldots x_{i_{k}}^{s_{k}}=0$ для всех $k, i_{2}, \ldots, i_{k}$; $s_{1}, \ldots, s_{k}$. Теорема доказана.

Эта теорема означает следующее: любой многочлен $g \in M(P)$ однозначно представляется в виде

$$
g=a_{0}+\sum_{1 \leqslant k, 1 \leqslant i_{2}<\ldots<i_{k}, 1 \leqslant s_{j} \leqslant q-1} l_{i_{2}, \ldots, i_{k} ; s_{1}, \ldots, s_{k}} \triangleleft x_{i_{1}}^{s_{1}} \ldots x_{i_{k}}^{s_{k}},
$$

где $a_{0} \in P, l_{i_{2}, \ldots, i_{k} ; s_{1}, \ldots, s_{k}} \in L(P)$. Это представление назовем разложением многочлена по одночленам с первой переменной. Его легко получить, используя (1).

В качестве следствия из теоремы 5 получаем теорему об аффинных левых делителях. 
Теорема 6. Пусть $g, g_{1}, g_{2} \in M(P)$ и разложения этих многочленов по одночленам $c$ первой переменной имеют вид

$$
\begin{aligned}
g & =a_{0}+\sum_{1 \leqslant k, 1 \leqslant i_{2}<\ldots<i_{k}, 1 \leqslant s_{j} \leqslant q-1} l_{i_{2}, \ldots, i_{k} ; s_{1}, \ldots, s_{k}} \triangleleft x_{i_{1}}^{s_{1}} \ldots x_{i_{k}}^{s_{k}}, \\
g_{1} & =a_{0}^{\prime}+\sum_{1 \leqslant k, 1 \leqslant i_{2}<\ldots<i_{k}, 1 \leqslant s_{j} \leqslant q-1} l_{i_{2}, \ldots, i_{k} ; s_{1}, \ldots, s_{k}}^{\prime} \triangleleft x_{i_{1}}^{s_{1}} \ldots x_{i_{k}}^{s_{k}}, \\
g_{2} & =a_{0}^{\prime \prime}+l,
\end{aligned}
$$

где $l \in L(P)$. Тогда следующие утверждения равносильнь:

(1) $g=g_{2} \triangleleft g_{1}$,

(2) $l_{i_{2}, \ldots, i_{k} ; s_{1}, \ldots, s_{k}}=l \triangleleft l_{i_{2}, \ldots, i_{k} ; s_{1}, \ldots, s_{k}}^{\prime}$ для всех $1 \leqslant k, 1<i_{2}<\ldots<i_{k}, 1 \leqslant s_{j} \leqslant q-1 u$ $a_{0}=a_{0}^{\prime \prime}+l\left(a_{0}^{\prime}, \ldots, a_{0}^{\prime}\right)$,

(3) $v^{-1}\left(l_{i_{2}, \ldots, i_{k} ; s_{1}, \ldots, s_{k}}\right)=v^{-1}(l) v^{-1}\left(l_{i_{2}, \ldots, i_{k} ; s_{1}, \ldots, s_{k}}^{\prime}\right)$ для всех $1 \leqslant k, 1<i_{2}<\ldots<i_{k}$, $1 \leqslant s_{j} \leqslant q-1$ u $a_{0}=a_{0}^{\prime \prime}+l\left(a_{0}^{\prime}, \ldots, a_{0}^{\prime}\right)$.

Утверждение (3) этой теоремы сводит задачу об описании всех разложений $g=g_{2} \triangleleft g_{1}$ с аффинным многочленом $g_{2}$ к задаче об описании всех общих делителей многочленов $v^{-1}\left(l_{i_{2}, \ldots, i_{k} ; s_{1}, \ldots, s_{k}}\right) \in P[x]$.

Если $g=g_{2} \triangleleft g_{1}, g_{2}=a+a_{i} x_{i}, 1 \leqslant i, a, a_{i} \in P$, то $g_{2}$ назовем тривиальным левым делителем многочлена $g$.

Для любого ненулевого многочлена $g \in M(P)$ через $d^{\prime}(g)$ обозначим максимальное число переменных во входящих в $g$ одночленах. Для двоичного случая $d^{\prime}(g)=d(g)$.

Следствие 3. Пусть $g \in M(P), d(g) \geqslant 2$ и выполнено хотя бы одно из следующих условии:

(1) для некоторого $s \geqslant 1$ в $g$ входит только один одночлен степени $s$,

(2) для некоторого $k_{0} \geqslant 1$ в g входит только один одночлен с числом переменных $k_{0}$,

(3) для некоторого $r$, некратного $q$, в $g$ входит только один одночлен с номером вида $q^{t} r$,

(4) $g=\sum_{0 \leqslant k, 1 \leqslant i_{1}<\ldots<i_{k}, 1 \leqslant s_{j} \leqslant q-1} a_{i_{1}, \ldots, i_{k} ; s_{1}, \ldots, s_{k}} x_{i_{1}}^{s_{1}} \ldots x_{i_{k}}^{s_{k}}, a_{n+1 ; 1} \neq 0, d^{\prime}(g) \geqslant 2 u$ многочлен $\sum_{i=1}^{n+1} a_{i ; 1} x^{i-1}$ неприводим.

Тогда g не имеет неприводимых аффинных левых делителей.

Доказательство. Из (1) следует (3) и из (2) следует (3).

Пусть выполнено (3), и этот одночлен есть $x_{i_{1}}^{s_{1}} \ldots x_{i_{k_{0}}}^{s_{k_{0}}}$ (то есть $t=i_{1}-1$ ). Тогда в обозначениях теоремы $6 l_{i_{2}-i_{1}+1, \ldots, i_{k_{0}-i_{1}+1} ; s_{1}, \ldots, s_{k_{0}}}=a_{i_{1}, \ldots, i_{k_{0}} ; s_{1}, \ldots, s_{k_{0}}} x_{i_{1}}$ и $l=a_{i} x_{i}$ для некоторого $1 \leqslant i \leqslant i_{1}, a_{i} \neq 0$.

Пусть выполнено условие (4). Тогда в обозначениях теоремы 6 многочлен $v^{-1}\left(l_{1}\right)=\sum_{i=1}^{n+1} a_{i ; 1} x^{i-1}$ неприводим, и следовательно, либо $v^{-1}(l)=a \neq 0$, либо $v^{-1}(l)=v^{-1}\left(l_{1}\right)$.

Если в многочлен $g$ входит член вида $x_{i_{1}}^{s_{1}} \ldots x_{i_{k}}^{s_{k}}$, то $i_{1} \leqslant n-k+2$. Следовательно, в формуле (2)

$$
d\left(v^{-1}\left(l_{i_{2}, \ldots, i_{k} ; s_{1}, \ldots, s_{k}}\right)\right) \leqslant n-k+1 .
$$


Допустим, что $v^{-1}(l)=v^{-1}\left(l_{1}\right)$. Из неприводимости этого многочлена и теоремы 6 следует, что тогда $l_{i_{2}, \ldots, i_{k} ; s_{1}, \ldots, s_{k}}=0$ для всех $k \geqslant 2$. Тогда из формулы (2) следует, что $d^{\prime}(g) \leqslant 1$, получаем противоречие. Следствие доказано.

Следствие 4. Пусть $f \in F_{n+1}^{*}(P), f$ существенно зависит от первой переменной и для представляющего функиию $f^{-1}$ многочлена $g$ выполнено условие следствия 3. Тогда регистр $R(f)$ не имеет нетривиальных внутренних гомоморфизмов в линейные автоматы над полем $P$ с одномерным входом без выхода.

Доказательство. Пусть $\beta: R(f) \rightarrow B$ - гомоморфизм в линейный автомат над полем $P$ с одномерным входом без выхода. Тогда по теореме 3 существуют функции $f_{1} \in F_{n_{1}+1}^{*}(P)$, $f_{2} \in F_{n_{2}+1}^{*}(P)$, где $n_{1}+n_{2}=n$, такие, что $f^{-1}=f_{2} \triangleleft f_{1}, f_{2}-$ унитарная аффинная функция, $\beta(R(f)) \cong R\left(f_{2}^{-1}\right)$. Тогда $f_{2}=a+x_{n_{2}+1}$ по следствию 3 . Поскольку и $f^{-1}$ существенно зависит от первой переменной, справедливо равенство $n_{2}=0$, и следствие доказано.

Для двоичного случая следствие 4 приобретает более простой вид.

Следствие 5. Пусть $P=G F(2), f \in F_{n+1}^{*}(P), f$ существенно зависит от первой переменной, $g=\sum_{0 \leqslant k, 1 \leqslant i_{1}<\ldots<i_{k} \leqslant n+1} x_{i_{1}} \ldots x_{i_{k}}$ - представляющий функцию $f$ многочлен, $d(g) \geqslant 2$, и выполнено хотя бы одно из следующих условий:

(1) для некоторого $s \geqslant 1$ в g входит только один одночлен степени $s$,

(2) для некоторого нечетного $r$ в $g$ входит только один одночлен с номером вида $2^{t} r$,

(3) многочлен $\sum_{i=1}^{n+1} a_{i} x^{i-1}$ неприводим.

Тогда регистр $R(f)$ не имеет нетривиальных гомоморфизмов в линейньле автомать над полем Р с одномерным входом без выхода.

Доказательство. Пусть $u(\alpha, \beta): R(f) \rightarrow B-$ гомоморфизм в линейный автомат над полем $P$ с одномерным входом без выхода. Тогда $\beta: R(f) \rightarrow B \alpha-$ внутренний гомоморфизм в линейный автомат и по следствию 4 он тривиален. Следствие доказано.

Следствие 6. Пусть $f \in F_{n+1}^{*}(P), f_{2} \in F_{n_{2}+1}^{*}(P), f_{2}-$ унитарная аффинная функция и регистр $R\left(f_{2}\right)$ является внутренне гомоморфнылм образом регистра $R(f)$. Тогда $n_{2} \leqslant n-d^{\prime}(g)+1$, где $g-$ многочлен, представляющий функцию $f^{-1}$.

Доказательство. По теореме $1, g=g_{2} \triangleleft g_{1}$, где $g_{2}=a_{0}^{\prime \prime}+l-$ аффинный многочлен $d\left(v^{-1}(l)\right)=n_{2}$. В разложении (2) при $k=d^{\prime}(g)=n_{2}$ есть коэффициент $l_{i_{2}, \ldots, i_{k} ; s_{1}, \ldots, s_{k}}$, отличный от нуля. По теореме 6 и формуле (3),

$$
n_{2}=d\left(v^{-1}(l)\right) \leqslant d\left(v^{-1}\left(l_{i_{2}, \ldots, i_{k} ; s_{1}, \ldots, s_{k}}\right)\right) \leqslant n-d^{\prime}(g)+1 .
$$

Следствие доказано.

Для двоичного случая эту оценку можно уточнить.

Следствие 7. Пусть $P=G F(2), f \in F_{n+1}^{*}(P), f_{2} \in F_{n_{2}+1}^{*}(P), d(f) \geqslant 2, f_{2}-a \phi$ финная функиия и регистр $R\left(f_{2}\right)$ является внутренне гомоморфным образом регистра $R(f)$. Тогда $n_{2} \leqslant n-d(f)$. 
Доказательство. По теореме $1, g=g_{2} \triangleleft g_{1}$, где $g_{2}=a_{0}^{\prime \prime} \oplus l-$ аффинный многочлен, $d\left(v^{-1}(l)\right)=n_{2}$. Поскольку $f$ линейна по последней переменной, в разложении (2) при $k \geqslant 2$

$$
d\left(v^{-1}\left(l_{i_{2}, \ldots, i_{k}}\right)\right) \geqslant n-k .
$$

В разложении (2) при $k=d(g)$ есть $l_{i_{2}, \ldots, i_{k}} \neq 0$. По теореме 6 и формуле (4),

$$
n_{2}=d\left(v^{-1}(l)\right) \leqslant d\left(v^{-1}\left(l_{i_{2}, \ldots, i_{k}}\right)\right) \leqslant n-d(g) .
$$

Следствие доказано.

Пример 1 (двоичный случай). Опишем все гомоморфизмы регистра $R(f)$ на линейные регистры для функции $f \in F_{n+1}^{*}(G F(2))$, где $n=8$ и $f$ представляется многочленом

$$
g=x_{1} \oplus x_{2} \oplus x_{3} \oplus x_{7} \oplus x_{8} \oplus x_{9} \oplus x_{2} x_{4} \oplus x_{3} x_{5} \oplus x_{3} x_{6} \oplus x_{4} x_{6} \oplus x_{4} x_{7} \oplus x_{5} x_{8} .
$$

1. По формуле (1) найдем разложение $g$ по одночленам с первой переменной:

$$
g=\left(x_{1} \oplus x_{2} \oplus x_{3} \oplus x_{7} \oplus x_{8} \oplus x_{9}\right) \triangleleft x_{1} \oplus\left(x_{2} \oplus x_{3} \oplus x_{4}\right) \triangleleft x_{1} x_{3} \oplus\left(x_{3} \oplus x_{4} \oplus x_{5}\right) \triangleleft x_{1} x_{4} .
$$

2. Справедливы равенства

$$
\begin{aligned}
v^{-1}\left(l_{\varnothing}\right) & =1 \oplus x \oplus x^{2} \oplus x^{6} \oplus x^{7} \oplus x^{8} \\
& =\left(1 \oplus x \oplus x^{2}\right)\left(1 \oplus x^{6}\right), \\
v^{-1}\left(l_{3}\right) & =x \oplus x^{2} \oplus x^{3}=\left(1 \oplus x \oplus x^{2}\right) x, \\
v^{-1}\left(l_{4}\right) & =x^{2} \oplus x^{3} \oplus x^{4}=\left(1 \oplus x \oplus x^{2}\right) x^{2},
\end{aligned}
$$

и следовательно, многочлен $1 \oplus x \oplus x^{2}-$ единственный нетривиальный общий делитель этих многочленов.

3. Для $a=0,1$ положим

$$
\begin{aligned}
g_{2, a} & =a \oplus x_{1} \oplus x_{2} \oplus x_{3}, \\
g_{1, a} & =a \oplus\left(x_{1} \oplus x_{7}\right) \triangleleft x_{1} \oplus x_{2} \triangleleft x_{1} x_{3} \oplus x_{3} \triangleleft x_{1} x_{4} \\
& =a \oplus x_{1} \oplus x_{7} \oplus x_{2} x_{4} \oplus x_{3} x_{6} .
\end{aligned}
$$

Тогда по теореме $6 g=g_{2, a} \triangleleft g_{1, a}, a=0,1,-$ все нетривиальные разложения с левым аффинным многочленом.

4. Для $a=0,1$ положим

$$
\begin{array}{ll}
f_{2, a} \in F_{3}^{*}(G F(2)), & f_{2, a}=g_{2, a}, \\
f_{1, a} \in F_{7}^{*}(G F(2)), & f_{1, a}=g_{1, a} .
\end{array}
$$

Тогда по теореме 3

$$
\left(x \oplus a_{0}, \pi_{f_{1, a}, 2}\right): R\left(f_{2, a} \oplus a_{0}\right), \quad a, a_{0}=0,1,
$$

- все гомоморфизмы регистра $R(f)$ на линейные регистры ненулевой длины. 


\section{Список литературы}

1. Башев В. А., Теоретико-групповая характеризация неавтономных линейных регистров сдвига. Труды по дискретной математике (2004) 8, 52-68.

2. Солодовников В. И., Гомоморфизмы двоичных регистров сдвига. Дискретная математика (2005) 17, №1, 73-88.

Статья поступила 24.12.2006. 